J. Korean Math. Soc. 52 (2015), No. 5, pp. 929-944

http://dx.doi.org/10.4134/JKMS.2015.52.5.929

\title{
SOME POLYNOMIAL INVARIANTS OF WELDED LINKS
}

\author{
Young Ho Im, Kyeonghui Lee, And Mi Hwa Shin
}

\begin{abstract}
We give a quotient of the ring $\mathbb{Q}\left[A^{ \pm 1}, t^{ \pm 1}\right]$ so that the Miyazawa polynomial is a non-trivial invariant of welded links. Furthermore we show that this is also an invariant under the other forbidden move $F_{u}$, and so it is a fused isotopy invariant. Also, we give some quotient ring so that the index polynomial can be an invariant for welded links.
\end{abstract}

\section{Introduction}

Virtual links were introduced by L. H. Kauffman [11] as a generalization of classical knot theory in the sense that two classical link diagrams are equivalent as virtual links, then they are equivalent as classical links [3, 11]. A virtual link diagram is an oriented link diagram in $\mathbb{R}^{2}$ possibly with some encircled crossings without over/under information, called virtual crossings. A virtual link is the equivalence class of such a link diagram by virtual isotopy (respectively, regular virtual isotopy), which consists of (classical) Reidemeister moves of type $R_{1}, R_{2}$ and $R_{3}$ (respectively, $R_{2}$ and $R_{3}$ ) and virtual Reidemeister moves of type $V R_{1}, V R_{2}, V R_{3}$ and the semivirtual move $V R_{4}$ as shown in Figure 1. However the forbidden moves $F_{o}$ and $F_{u}$ shown in Figure 2 are not allowed.

Welded isotopy (respectively, regular welded isotopy) is the extension of virtual isotopy (respectively, regular virtual isotopy) which also allows the $F_{o}$ moves. A welded link is the equivalence class of a virtual link diagram under welded isotopy, and welded links can be obtained as the closure of welded braids. See $[2,7]$ for more details.

As applications to surface knots, S. Satoh [14] has shown that there is an algorithm to produce ribbon torus knots from welded knot diagrams via the

Received October 23, 2014; Revised January 30, 2015.

2010 Mathematics Subject Classification. Primary 57M25; Secondary 57M27.

Key words and phrases. virtual link, welded link, Miyazawa polynomial, index polynomial, fused isotopy.

The first author was supported by Basic Science Research Program through the National Research Foundation of Korea(NRF) funded by the Ministry of Education, Science and Technology(NRF-2014R1A1A2055060) and the second author was supported by Basic Science Research Program through the National Research Foundation of Korea(NRF) funded by the Ministry of Science, ICT \& Future Planning(NRF-2014R1A1A1002317). 


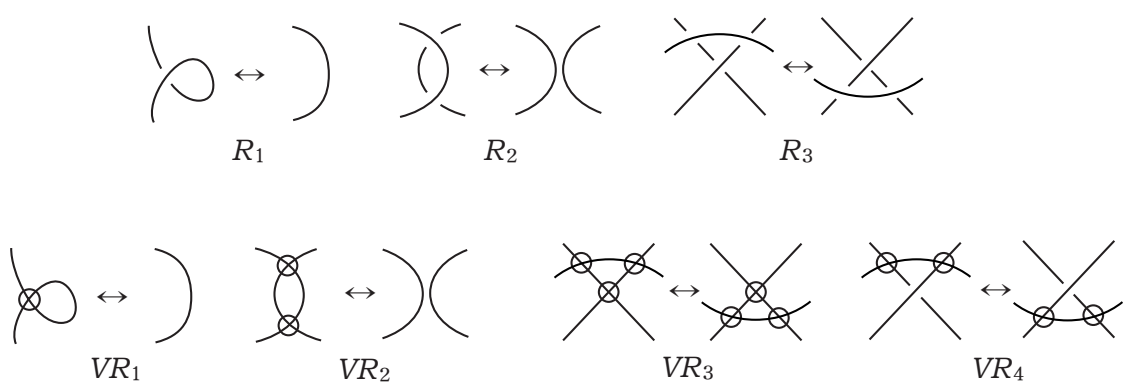

Figure 1. Virtual isotopy



$F_{O}$

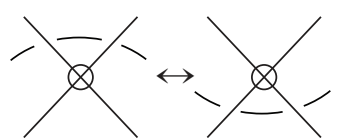

$F_{u}$

Figure 2. Forbidden moves

operation known as Tube. Furthermore, this operation was shown to be independent of the particular representative of a welded equivalence class of welded knots. However, ribbon torus knots are not classified by welded knots under the Tube operation. B. Winter [15] gave a specific example of inequivalent welded knots which are mapped to the same ribbon torus knot by the Tube operation, and he also showed that if two classical knot diagrams are welded equivalent, then they are equivalent as classical knots as well.

It is worth noting that the knot group and Alexander polynomial are welded isotopy invariant and so any knot with non-trivial knot group or non-trivial Alexander polynomial is not welded isotopic to the unknot. Therefore, the class of welded knots is not trivial. Allowing both of the forbidden moves $F_{o}$ and $F_{u}$ gives rise to fused isotopy introduced by Kauffman in [11]. It was shown in [10] and [13] that any virtual knot is fused isotopic to the unknot.

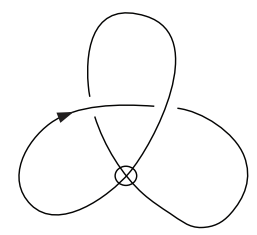

Figure 3. Virtual trefoil knot 
The virtual trefoil knot in Figure 3 is not virtually the unknot by the nontrivial Jones-Kauffman polynomial. However, it is the unknot under welded isotopy. This implies that the Jones-Kauffman polynomial defined in the ring $\mathbb{Z}\left[q^{ \pm \frac{1}{4}}\right]$ is not an invariant of welded links. As a consequence, A. Fish and E. Keyman [1] considered a quotient of the $\operatorname{ring} \mathbb{Z}\left[q^{ \pm \frac{1}{4}}\right]$ which is $\mathbb{Z}\left[q^{ \pm \frac{1}{4}}\right] / I$ for some ideal $I$, so that the Jones-Kauffman polynomial is a non-trivial isotopy invariant of welded links under this quotient ring.

In this paper, we give a quotient of the ring $\mathbb{Q}\left[A^{ \pm 1}, t^{ \pm 1}\right]$ so that the Miyazawa polynomial introduced in [12] is a non-trivial invariant of welded links. Furthermore, we show that this is also an invariant under the other forbidden move $F_{u}$, and so it is a fused isotopy invariant. Also, we give some quotient ring so that the index polynomial introduced in [5] can be a non-trivial invariant for welded links.

\section{The Miyazawa polynomial for welded links}

In this section, we consider the Miyazawa polynomials for welded links.

\subsection{The Miyazawa polynomial $M_{D}(A, t)$ for welded links}

The Miyazawa polynomial of oriented virtual links was introduced in [12], which takes values in the ring $\mathbb{Q}\left[A^{ \pm 1}, t^{ \pm 1}\right]$. We first review briefly the polynomial [8] which is shown to be equivalent to the Miyazawa polynomial and then investigate its behavior for welded links.

A magnetic graph in the 3 -sphere $S^{3}$ is a 2 -valent graph $G$ with vertices in $S^{3}$ such that the edges of $G$ are oriented alternately as in Figure 4 . We allow $G$ to have components consisting of closed edges without vertices.

Figure 4. Magnetic graph

A magnetic graph diagram is a projection image on a plane equipped with over/under information on each crossing.

A virtual magnetic graph diagram, which is written as VMG diagram for short, is a magnetic graph diagram possibly with some virtual crossings. See Figure 5. Crossings that are not encircled are called real crossings. If two VMG diagrams are related by a finite sequence of generalized Reidemeister moves, they are said to be equivalent.

Let $D$ be a VMG diagram, and let $p$ be a real crossing. By $A$-splice (or $B$-splice) at $p$, we mean the local replacement about $p$ depicted in Figure 6 .

A state of $D$ is a VMG diagram obtained from $D$ by doing $A$-splice or $B$ splice at each real crossing. Let $S$ be a state of a VMG diagram of $D$. A weight map $\sigma$ of $S$ is a map from the set of edges of $S$ to $\{+1,-1\}$ such that $\sigma(e) \neq \sigma\left(e^{\prime}\right)$ for adjacent edges $e$ and $e^{\prime}$ of $S$. An enhanced state means a pair 


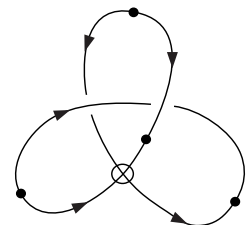

Figure 5. A VMG diagram

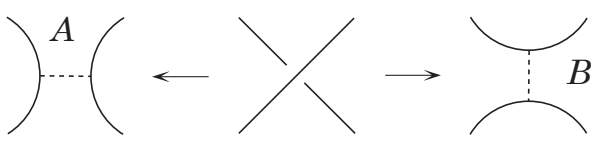

Figure 6. A-splice and B-splice

$(S, \sigma)$ of a state $S$ and a weight map $\sigma$ of $S$. The set of enhanced states of $D$ is denoted by $s(D)$.

Let $(S, \sigma)$ be an enhanced state of a VMG diagram $D$. For a virtual crossing $v$ of $S$ on edges $e$ and $e^{\prime}$ as depicted in Figure 7(1), the sign of $v$ is defined by $\frac{\sigma(e)-\sigma\left(e^{\prime}\right)}{2}$. See Figure $7(2)$, where edges assigned +1 by the weight map $\sigma$ are drawn thickly. The virtual writhe $\omega^{v}(S, \sigma)$ of $(S, \sigma)$ is defined to be the sum of signs of all virtual crossings.

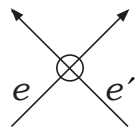

$(1)$

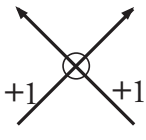

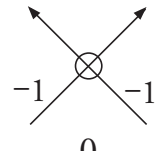

0

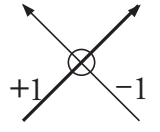

$+1$

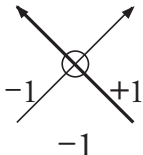

$-1$

(2)

FiguRE 7. Sign of virtual crossing $v$

For a VMG diagram $D$, we define $\ll D \gg_{M} \in \mathbb{Q}\left[A^{ \pm 1}, t^{ \pm 1}\right]$ by

$$
\ll D \gg_{M}=\sum_{(S, \sigma) \in s(D)} 2^{-\# S} A^{\natural S}\left(-A^{2}-A^{-2}\right)^{\# S-1} t^{\omega^{v}(S, \sigma)},
$$

where $\sharp S$ is the number of $A$-splices minus that of $B$-splices used to obtain $S$ from $D$, and $\# S$ is the number of components of $S$. The Miyazawa polynomial of $D$, which we denote by $M_{D}(A, t)$, is defined by

$$
M_{D}(A, t)=\left(-A^{3}\right)^{-\omega(D)} \ll D \gg_{M},
$$

where $\omega(D)$, called the writhe, is the number of positive crossings minus that of negative crossings of $D$. 
Theorem 2.1 ([8]). The Miyazawa polynomial $M_{D}(A, t)$ is an invariant of the equivalence class of a VMG diagram.

Now, we investigate the Miyazawa polynomial for welded links. We begin with the following definition.

Definition 2.2. Let $D$ be a virtual link diagram and let $B^{2}$ be a disc. $T=$ $D \cap B$ is called a (generalized) tangle if $D$ intersects $\partial B^{2}$ transversally and $D \cap \partial B^{2}$ does not contain any crossing points.

Let $T$ and $P$ be two oriented tangles which agree on the boundary of the disc $B$ as shown in Figure 8. Consider all possible ways of joining the end points of $\partial B$ outside $B$ without introducing any real crossings. According to the orientation of $T$, each joining gives VMG diagrams $D_{T}, D_{P}$, which are obtained from $T$ and $P$ by connecting the end points of $\partial B$ outside $B$ without real crossings and are identical outside $B$. Then $D_{P}$ is obtained by an $F_{o}$ move from the VMG diagram $D_{T}$. We will compute $\ll D_{T} \gg_{M}-\ll D_{P} \gg_{M}$. Let $I_{1}(A, t)$ be the ideal of $\mathbb{Q}\left[A^{ \pm 1}, t^{ \pm 1}\right]$ generated by these polynomials.
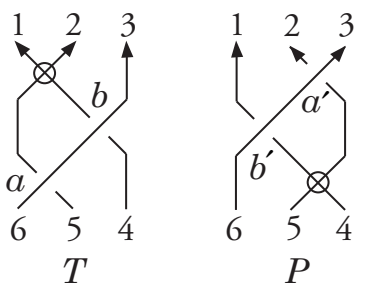

Figure 8 . Tangles $T$ and $P$



Figure 9. States of $T$ and $P$

In Figure 9, each $T_{i}$ and $P_{i}$ can have vertices according to the orientation. Outside $B$, there are 15 ways of connecting the end points of $T$ or $P$. Then, we obtain the following Table 1 by computing $\ll D_{T} \gg_{M}-\ll D_{P} \gg_{M}$. 
Table 1

\begin{tabular}{|c|l|}
\hline outside connection & $\ll D_{T} \gg_{M}-\ll D_{P} \gg_{M}$ \\
\hline$[12][34][56]$ & 0 \\
\hline$[12][35][46]$ & 0 \\
\hline$[12][36][45]$ & 0 \\
\hline$[13][24][56]$ & $\left(A^{4}-1\right)+\left(\frac{t+t^{-1}}{2}\right)\left(A^{-6}-A^{-2}\right)$ \\
\hline$[13][25][46]$ & 0 \\
\hline$[13][26][45]$ & 0 \\
\hline$[14][23][56]$ & 0 \\
\hline$[14][25][36]$ & 0 \\
\hline$[14][26][35]$ & 0 \\
\hline$[15][23][46]$ & $-\left(A^{4}-1\right)-\left(\frac{t+t^{-1}}{2}\right)\left(A^{-6}-A^{-2}\right)$ \\
\hline$[15][24][36]$ & 0 \\
\hline$[15][26][34]$ & $\left(A^{6}-A^{2}\right)+\left(\frac{t+t^{-1}}{2}\right)\left(A^{-4}-1\right)$ \\
\hline$[16][23][45]$ & 0 \\
\hline$[16][24][35]$ & $-\left(A^{6}-A^{2}\right)-\left(\frac{t+t^{-1}}{2}\right)\left(A^{-4}-1\right)$ \\
\hline$[16][25][34]$ & 0 \\
\hline
\end{tabular}

Let $g(A, t)=\left(A^{6}-A^{2}\right)+\left(\frac{t+t^{-1}}{2}\right)\left(-1+A^{-4}\right)$. Then $A^{-2} g(A, t)=\left(A^{4}-1\right)+$ $\left(\frac{t+t^{-1}}{2}\right)\left(-A^{-2}+A^{-6}\right)$.

Similarly, the other cases of $T$ and $P$ with different orientations can give $\ll D_{T} \gg_{M}-\ll D_{P} \gg_{M}= \pm g\left(A^{-1}, t\right)$ and $\pm A^{2} g\left(A^{-1}, t\right)$ by a simple calculation.

Then $I_{1}(A, t)$ is the ideal generated by two polynomials $g(A, t)=\left(A^{6}-\right.$ $\left.A^{2}\right)+\left(\frac{t+t^{-1}}{2}\right)\left(-1+A^{-4}\right)$ and $g\left(A^{-1}, t\right)=\left(A^{-6}-A^{-2}\right)+\left(\frac{t+t^{-1}}{2}\right)\left(-1+A^{4}\right)$, and we obtain the following result.

Theorem 2.3. If $D_{1}$ and $D_{2}$ are regular welded isotopic virtual link diagrams, then $\ll D_{1} \gg_{M} \equiv \ll D_{2} \gg_{M} \bmod I_{1}(A, t)$.

Proof. From the above calculations for all cases, we have

$\ll D_{T} \gg_{M}-\ll D_{P} \gg_{M} \in\left\{0, \pm g(A, t), \pm A^{-2} g(A, t), \pm g\left(A^{-1}, t\right), \pm A^{2} g\left(A^{-1}, t\right)\right\}$.

Therefore, the result follows.

Let $R$ denote the quotient ring $\mathbb{Q}\left[A^{ \pm 1}, t^{ \pm 1}\right] / I_{1}(A, t)$.

Corollary 2.4. The Miyazawa polynomial $M_{D}(A, t)$ of an oriented welded link diagram $D$ in $R$ is a welded isotopy invariant. 
Proof. Since an $F_{o}$ move does not change the writhe of the diagram, the result follows.

\subsection{The Miyazawa polynomial $R_{D}(A, t)$ with $t^{2}=1$ for welded links}

We consider the Miyazawa polynomial $M_{D}(A, t)$ which takes values in the quotient ring $\mathbb{Q}\left[A^{ \pm 1}, t\right] /\left(t^{2}-1\right)$. Then we have the following.

Lemma $2.5([6])$. Let $D$ be a virtual link diagram and $s$ be a state of $D$. Then the value $t^{\omega^{v}(s, \sigma)}$ in $M_{D}(A, t)$ does not depend on the choice of the weight map $\sigma$ in the quotient ring $\mathbb{Q}\left[A^{ \pm 1}, t\right] /\left(t^{2}-1\right)$, where $\omega^{v}(s, \sigma)$ is the virtual writhe of an enhanced state $(s, \sigma)$.

Because of Lemma 2.5, we shall regard an enhanced state $(s, \sigma)$ as the state $s$. It is easy to see that the Miyazawa polynomial $M_{D}(A, t)$ in $\mathbb{Q}\left[A^{ \pm 1}, t\right] /\left(t^{2}-1\right)$ is the same as $R_{D}(A, t)[9]$ which takes values in the quotient ring $\mathbb{Z}\left[A^{ \pm 1}, t\right] /\left(t^{2}-\right.$ $1)$ because for a state $s$ of $D i(s)=1$ if and only if $\omega^{v}(s) \equiv 0(\bmod 2)$ holds.

Recall the definition of the 2 -variable polynomial $R_{D}(A, t)$. When a weight map $\sigma$ is given, for a virtual crossing $v$ of $D$ where edges $e$ and $e^{\prime}$ intersect, the sign of $v$ with respect to $\sigma$ is defined to be the product of two weights $\sigma(e)$ and $\sigma\left(e^{\prime}\right)$ and denoted by $i_{\sigma}(v) . i(D)$ is defined to be the product of $i_{\sigma}(v)$ 's over all virtual crossings. Then $H_{D}(A, t)$ and $R_{D}(A, t)$ are defined for a VMG diagram $D$ as

$$
H_{D}(A, t)=\sum_{s \in \mathcal{S}(D)} A^{\alpha(s)-\beta(s)}\left(-A^{2}-A^{-2}\right)^{\gamma(s)-1} t^{(1-i(s)) / 2}
$$

and

$$
R_{D}(A, t)=\left(-A^{3}\right)^{-\omega(D)} H_{D}(A, t)
$$

where $S(D)$ is the set of states of $D, \alpha(s)$ (respectively, $\beta(s)$ ) is the number of A-splices (respectively, B-splices) and $\gamma(s)$ is the number of components of a state $s$.

Now, we investigate the Miyazawa polynomial $M_{D}(A, t)$ for welded links in the quotient ring $\mathbb{Z}\left[A^{ \pm 1}, t\right] /\left(t^{2}-1\right)$.

Let $I_{o}$ be the principal ideal generated by $g(A, t)=A^{6}-A^{2}+t\left(-1+A^{-4}\right)$ in the quotient ring $\mathbb{Z}\left[A^{ \pm 1}, t\right] /\left(t^{2}-1\right)$. Then we obtain the following result.

Theorem 2.6. If $D$ and $D^{\prime}$ are regular welded isotopic virtual link diagrams, then $H_{D}(A, t) \equiv H_{D^{\prime}}(A, t) \bmod I_{o}$.

Proof. We obtain Table 2 by computing $H_{D}(A, t)-H_{D^{\prime}}(A, t)$, and we have $H_{D}(A, t)-H_{D^{\prime}}(A, t) \in\left\{0, \pm g(A, t), \pm A^{-2} t g(A, t)\right\}$. Therefore, the result follows.

Let $R_{1}$ denote the quotient ring $\mathbb{Z}\left[A^{ \pm 1}, t\right] /\left(t^{2}-1\right)$.

Corollary 2.7. The polynomial $R_{D}(A, t)$ of an oriented welded link $D$ in the quotient ring $R_{1} / I_{o}$ is a welded isotopy invariant. 
Table 2

\begin{tabular}{|c|l|}
\hline outside connection & $H_{D_{T}}(A, t)-H_{D_{P}}(A, t)$ \\
\hline$[12][34][56]$ & 0 \\
\hline$[12][35][46]$ & 0 \\
\hline$[12][36][45]$ & 0 \\
\hline$[13][24][56]$ & $\left(A^{-6}-A^{-2}\right)+t\left(-1+A^{4}\right)$ \\
\hline$[13][25][46]$ & 0 \\
\hline$[13][26][45]$ & 0 \\
\hline$[14][23][56]$ & 0 \\
\hline$[14][25][36]$ & 0 \\
\hline$[14][26][35]$ & 0 \\
\hline$[15][23][46]$ & $-A^{-6}+A^{-2}+t\left(1-A^{4}\right)$ \\
\hline$[15][24][36]$ & 0 \\
\hline$[15][26][34]$ & $A^{6}-A^{2}+t\left(-1+A^{-4}\right)$ \\
\hline$[16][23][45]$ & 0 \\
\hline$[16][24][35]$ & $-A^{6}+A^{2}+t\left(1-A^{-4}\right)$ \\
\hline$[16][25][34]$ & 0 \\
\hline &
\end{tabular}

Proof. Since an $F_{o}$ move does not change the writhe of the diagram, the result follows.

Welded isotopy allows $F_{o}$ moves, but not $F_{u}$ moves. Let $T^{\prime}$ and $P^{\prime}$ be obtained from oriented tangles $T$ and $P$ in the Subsection 2.1 by changing all over crossings to under crossings, respectively. Note that the states of $T^{\prime}$ are the same as states of $T$, but we have $H_{D_{T}^{\prime}}(A, t)=H_{D_{T}}\left(A^{-1}, t\right)$. Then, we get $H_{D_{T}^{\prime}}(A, t)-H_{D_{P}^{\prime}}(A, t)$ by replacing $A$ by $A^{-1}$ in $H_{D_{T}}(A, t)-H_{D_{P}}(A, t)$. Therefore, if $I_{u}(A, t)$ is the ideal generated by $H_{D_{T}^{\prime}}(A, t)-H_{D_{P}^{\prime}}(A, t)$ 's for all possible $T^{\prime}$ and $P^{\prime}$, then $I_{o}(A, t)=I_{u}(A, t)$ holds.

Fused isotopy is the virtual isotopy which allow both $F_{o}$ moves and $F_{u}$ moves. A fused link is the equivalence class of a virtual link diagram under fused isotopy. Thus if we allow both $F_{o}$ moves and $F_{u}$ moves, we have the following.

Theorem 2.8. The Miyazawa polynomial $R_{D}(A, t)$ of fused links in the quotient ring $R_{1} / I_{o}$ is a fused isotopy invariant.

Proof. Since we have $I_{o}(A, t)=I_{u}(A, t)$, the result follows.

It is well known that any virtual knot is fused isotopic to the unknot $[10,13]$. Therefore, we get the following result. 
Theorem 2.9. The Miyazawa polynomial $R_{D}(A, t)$ of any virtual knot diagram $D$ in the quotient ring $R_{1} / I_{o}(A, t)$ is 1 .

Corollary 2.10. If $D$ is virtual knot diagram, then $H_{D}(A, t)$ is $(-A)^{3 w(D)}$ in the quotient ring $R_{1} / I_{o}(A, t)$.

Proof. For a virtual knot diagram $D, R_{D}(A, t) \equiv 1 \bmod I_{o}(A, t)$. Thus, $H_{D}(A, t) \equiv(-A)^{3 w(D)} \bmod I_{o}(A, t)$.

As an application, we can calculate the polynomial $R_{D}(A, t)$ in the quotient ring $R_{1}$ for any virtual link diagram $D$ with two components via the linking number of $D$.

Lemma 2.11 ([9]). Let $D$ be a VMG diagram and $p$ a real crossing on $D$. Then we have

$$
H_{D}(A, t)=A^{\operatorname{sign}(p)} H_{Z_{p} D}(A, t)+A^{-\operatorname{sign}(p)} H_{I_{p} D}(A, t),
$$

where the $\operatorname{sign}(p)$ is defined to be +1 on the left of Figure 10 and if the crossing is changed, then the $\operatorname{sign}(p)$ is defined to be -1 , and $Z_{p} D$ is a diagram from $D$ by doing A-splice (respectively, B-splice) at a positive (respectively, negative) real crossing $p$ and $I_{p} D$ is a diagram from $D$ by doing $B$-splice (respectively, $A$-splice) at a positive (respectively, negative) real crossing $p$ and appending two vertices as in Figure 10.
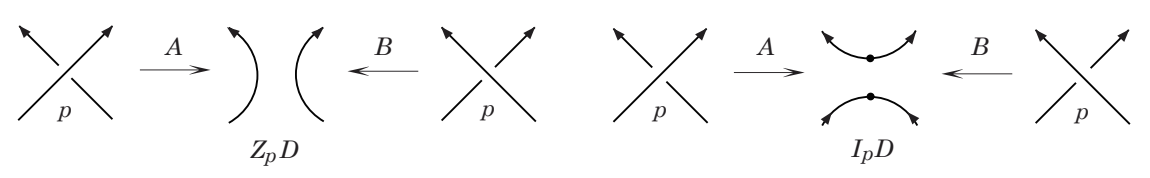

Figure 10. VMG diagrams $Z_{p} D$ and $I_{p} D$

Let $D$ be a VMG diagram, $B$ be a local disk in $\mathbb{R}^{2}$ and let the restriction of $D$ to $B$ be denoted by $\alpha$. Then $\alpha$ is an arc of $D$ which has neither self crossing nor vertex. If we divide $\alpha$ into three edges by putting two vertices $a$ and $b$ on $\alpha$ and reverse the orientation of the edge on $\alpha$, whose endpoints are $a$ and $b$, we obtain a new VMG diagram $D^{\prime}$. We say that $D^{\prime}$ is obtained from $D$ by the division of an edge as in Figure 11.



Figure 11. Division of an edge 
Lemma $2.12([9])$. Let $D$ and $D^{\prime}$ be VMG diagrams. If $D^{\prime}$ is obtained from $D$ by the division of an edge, then

$$
H_{D}(A, t)=H_{D}^{\prime}(A, t) .
$$

Proposition 2.13. Let $D$ be a virtual link diagram and $p$ a real crossing on $D$. If $D^{\prime}$ is obtained from $D$ by appending two vertices as shown in Figure 12, then $H_{D^{\prime}}(A, t)=H_{D}(A, t)$ and $R_{D^{\prime}}(A, t)=R_{D}(A, t)\left(-A^{-3}\right)^{-2 \operatorname{sign}(p)}$.

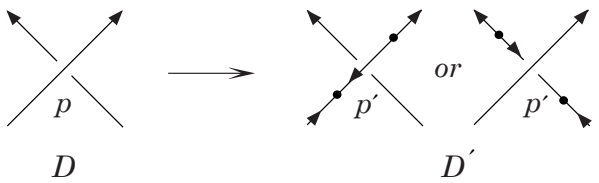

Figure 12. Append two vertices

Proof. By Lemma 2.11, we have

$$
H_{D}(A, t)=A^{\operatorname{sign}(p)} H_{Z_{p} D}(A, t)+A^{-\operatorname{sign}(p)} H_{I_{p} D}(A, t)
$$

and

$$
H_{D^{\prime}}(A, t)=A^{\operatorname{sign}\left(p^{\prime}\right)} H_{Z_{p^{\prime}} D^{\prime}}(A, t)+A^{-\operatorname{sign}\left(p^{\prime}\right)} H_{I_{p^{\prime}} D^{\prime}}(A, t) .
$$

(See Figure 13.)
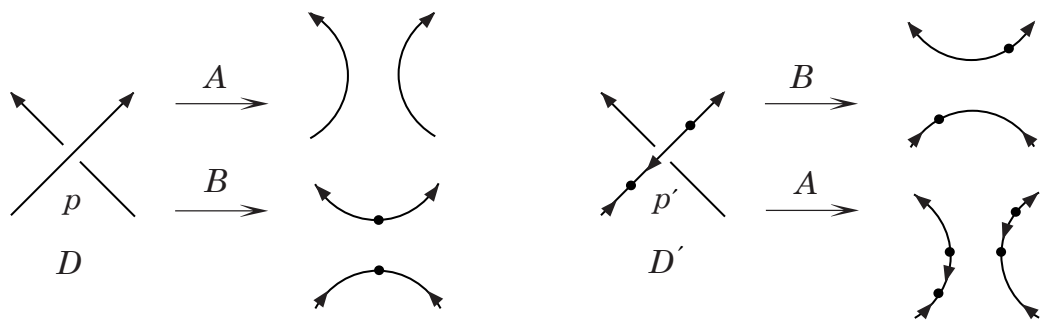

Figure 13. A-splice and B-splice at a positive crossing $p$

It is immediate that $\operatorname{sign}(p)=-\operatorname{sign}\left(p^{\prime}\right)$ and $H_{I_{p} D}(A, t)=H_{Z_{p^{\prime}} D^{\prime}}(A, t)$. From Lemma 2.12, we obtain $H_{Z_{p} D}(A, t)=H_{I_{p^{\prime}} D^{\prime}}(A, t)$, and hence $H_{D^{\prime}}(A, t)$ $=H_{D}(A, t)$.

Since $w\left(D^{\prime}\right)=w(D)-2 \operatorname{sign}(p)$,

$$
\begin{aligned}
R_{D^{\prime}}(A, t) & =\left(-A^{-3}\right)^{w\left(D^{\prime}\right)} H_{D^{\prime}}(A, t) \\
& =\left(-A^{-3}\right)^{w\left(D^{\prime}\right)} H_{D}(A, t) \\
& =\left(-A^{-3}\right)^{w(D)-2 \operatorname{sign}(p)} H_{D^{\prime}}(A, t) \\
& =R_{D}(A, t)\left(-A^{-3}\right)^{-2 \operatorname{sign}(p)} .
\end{aligned}
$$


Proposition 2.14. Let $D$ be a virtual link diagram. If $D^{\prime}$ is obtained from $D$ by appending two vertices as shown in Figure 14 , then $H_{D^{\prime}}(A, t)=H_{D}(A, t) t$ and $R_{D^{\prime}}(A, t)=R_{D}(A, t) t$.

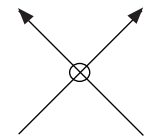

$D$

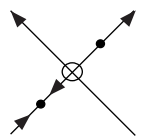

$D^{\prime}$

FiguRE 14

Proof. Let $S$ be any state of $D$. Then, there exists a unique state $S^{\prime}$ of $D^{\prime}$

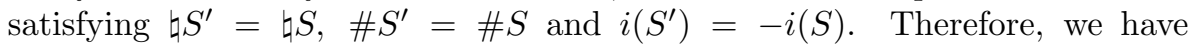
$H_{D^{\prime}}(A, t)=H_{D}(A, t) t$.

Since $w\left(D^{\prime}\right)=w(D)$, we obtain $R_{D^{\prime}}(A, t)=R_{D}(A, t) t$.

Theorem 2.15. Let $L=L_{1} \cup L_{2}$ be a virtual link with components $L_{1}$ and $L_{2}$. If the linking number of $L$ is $l k\left(L_{1}, L_{2}\right)=k$, then $R_{L}(A, t) \equiv-A^{-2}-A^{-12 k+2} t^{J}$ $\bmod I_{o}$ where $l k\left(L_{1}, L_{2}\right)$ is half the sum of the signs of the crossings at which one edge from $L_{1}$ and the other is from $L_{2}$, and $J \in\{0,1\}$ and $J \equiv 2 k \bmod 2$.

Proof. Let $D$ be a virtual link diagram of $L$ and let $D_{i}$ correspond to the component $L_{i}$ of $L$ for $i=1,2$. Then we obtain the writhe of $D, w(D)=$ $w\left(D_{1}\right)+w\left(D_{2}\right)+2 k$. Let $p$ be a real crossing between $D_{1}$ and $D_{2}$. Without

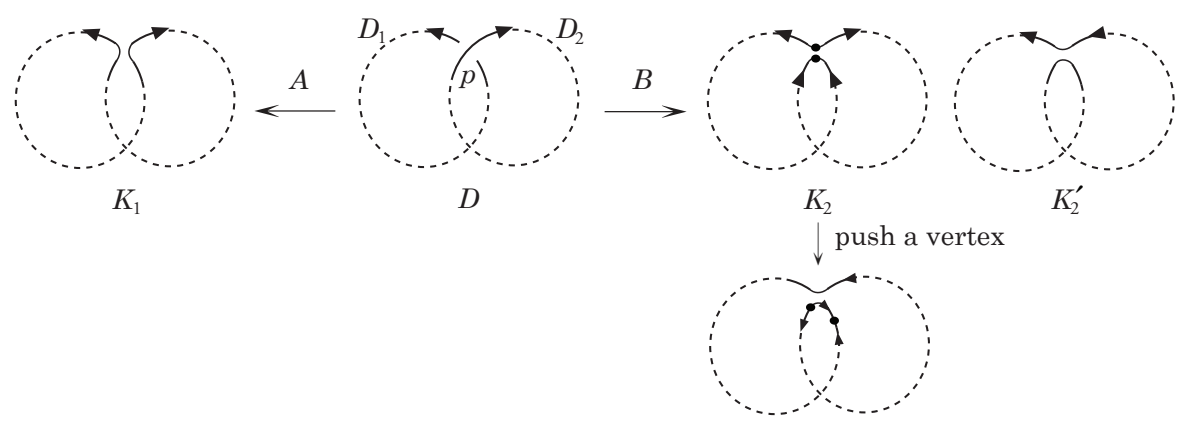

Figure 15. Diagrams by A-splice and B-splice

loss of generality, we assume $p$ is positive. By smoothing $D$ at $p$ as shown in Figure 15 , we have $H_{D}(A, t)=A H_{K_{1}}(A, t)+A^{-1} H_{K_{2}}(A, t)$.

$K_{1}$ and $K_{2}$ are diagrams obtained from $D$ by doing $A$-splice and $B$-splice at $p$, respectively. Let $K_{2}^{\prime}$ be the virtual knot diagram whose underlying set is $\left|K_{2}\right|$ without vertices so that the orientation is induced from $D_{1}$, as shown in 
Figure 15. Since the sum of the numbers of real and virtual crossings between $D_{1}$ and $D_{2}$ is the number of the crossings between $D_{1}$ and $D_{2}$ which is even, the numbers of real and virtual crossings between $D_{1}$ and $D_{2}$ have the same parity. We push a vertex of $K_{2}$ along the orientation of $D_{2}$ until two vertices are met as shown in Figure 15. From Propositions 2.13 and 2.14, we obtain that $H_{K_{2}}(A, t)=H_{K_{2}^{\prime}}(A, t)$ or $H_{K_{2}}(A, t)=H_{K_{2}^{\prime}}(A, t) t$ in the ring $\mathbb{Z}\left[A^{ \pm 1}, t\right] /\left(t^{2}-1\right)$ according to the number of all virtual crossings between $D_{1}$ and $D_{2}$ is even or odd. Therefore, $H_{K_{2}}(A, t) \equiv H_{K_{2}^{\prime}}(A, t) t^{J} \bmod I_{o}$, where $J \in\{0,1\}$ and $J \equiv 2 k$ $\bmod 2$. Since $w\left(K_{1}\right)=w(D)-1$ and $w\left(K_{2}^{\prime}\right)=w\left(D_{1}\right)+w\left(D_{2}\right)-(2 k-1)=$ $w(D)-4 k+1$, for $J \equiv 2 k \bmod 2$,

$$
\begin{aligned}
R_{D} & =(-A)^{-3 w(D)}\left(A H_{K_{1}}(A, t)+A^{-1} H_{K_{2}}(A, t)\right) \\
& \equiv(-A)^{-3 w(D)}\left(A H_{K_{1}}(A, t)+A^{-1} H_{K_{2}^{\prime}}(A, t) t^{J}\right) \\
& \equiv(-A)^{-3 w(D)}\left(A(-A)^{3 w\left(K_{1}\right)}+A^{-1}(-A)^{3 w\left(K_{2}^{\prime}\right)} t^{J}\right) \\
& \equiv-A^{-2}-A^{-12 k+2} t^{J} \quad \bmod I_{o} .
\end{aligned}
$$

\section{The index polynomial for welded links}

In this section we consider the index polynomial for welded links.

First we introduce an index polynomial for virtual links which is an extension of the polynomial invariant given in [4].

A real crossing of a virtual link diagram $D$ is said to be a self crossing if the crossing is composed of two arcs which belong to the same component of $D$. We denote the set of self crossings of $D$ by $S(D)$. A real crossing of $D$ is said to be a mixed crossing if the crossing is composed of two edges which belong to different components of $D$. We denote the set of mixed crossings of $D$ by $M(D)$. If we denote the set of real crossings of $D$ by $C(D)$, then it is obvious that $C(D)=S(D) \sqcup M(D)$.

Suppose that a real crossing $c$ of $D$ is a self crossing. Then, there exists a unique component of $D$ which constitutes $c$. We denote it by $D_{c}$. Let $\widehat{D_{c}}$ be a virtual link diagram obtained from $D_{c}$ by smoothing $c$ as shown in Figure 16 . If $c$ is a mixed crossing of $D$, then we define $\widehat{D_{c}}$ as the subdiagram of $D$ which consists of two components constituting $c$.



FiguRE 16. Smoothing operation

To define an index polynomial for virtual links, we choose an ordering $(1,2)$ for the components of the virtual link diagram $\widehat{D_{c}}$ as follows. For $c \in S(D)$, 
the over-arc containing $c$ of $D_{c}$ is the union of incoming arc $\alpha$ and outgoing arc $\beta$ at the shadow of $D_{c}$. Then $\widehat{D_{c}}$ is divided into two components containing $\alpha$ and $\beta$, respectively. We assign 1 (respectively, 2) for the component containing $\alpha$ (respectively, $\beta$ ). If a real crossing $c$ belongs to $M(D)$, then we choose an ordering $(1,2)$ for the two-component sublink diagram $\widehat{D_{c}}$ so that we assign 1 (respectively, 2) for the component containing the overarc (respectively, underarc).

Let $1 \cap 2$ denote the set of virtual crossings between the two components. For each virtual crossing $v \in 1 \cap 2$, we assign 1 or -1 , called the index number of $v$ and denoted by ind $(v)$ as shown in Figure 17. And for each virtual crossing $v$ in one component of $\widehat{D_{c}}$, we assign 0 .

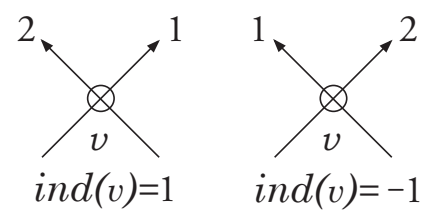

FiguRe 17. Index number

Definition 3.1. Let $D$ be a virtual link diagram and $c$ be a real crossing of $D$. Then the virtual intersection index, denoted by $i(c)$, of an ordered twocomponent virtual link diagram $\widehat{D_{c}}$ is given by

$$
i(c)=\sum_{v \in V\left(\widehat{D_{c}}\right)} i n d(v),
$$

where $V\left(\widehat{D_{c}}\right)$ is the set of virtual crossings of $\widehat{D_{c}}$.

With the fixed ordering of the components of $\widehat{D_{c}}$, we define a polynomial invariant for virtual links as follows.

Definition 3.2. Let $D$ be a virtual link diagram with $n$ components. Then the polynomial $Q_{D}(t) \in \mathbb{Z}\left[t^{ \pm 1}\right]$ for a virtual link diagram $D$ is defined by

$$
Q_{D}(t)=\sum_{c \in C(D)} \operatorname{sign}(c)\left(t^{i(c)}-1\right),
$$

where $C(D)$ is the set of all real crossings of $D$.

Theorem $3.3([5])$. The polynomial $Q_{D}(t) \in \mathbb{Z}\left[t^{ \pm 1}\right]$ is an invariant for a virtual link diagram $D$.

The index polynomial $Q_{D}(t)$ for a virtual link diagram $D$ introduced in [5] is not an invariant for welded links, as shown in the following example. 




FIGURE 18

Example 3.4. The virtual knot diagram $D$ in Figure 18, which is the unknot under welded isotopy, has non-trivial index polynomial $Q_{D}(t)=-\left(t^{-1}-1\right)-$ $(t-1)$.

We investigate how the index polynomials of virtual link diagrams can be changed under $F_{o}$ moves. Let $T$ and $P$ be two oriented tangles which agree on the boundary of the disc $B$ as shown in Figure 8. Consider all of possible ways of joining the end points of $\partial B$ outside $B$ so that they are virtual link diagrams. Each joining gives oriented virtual link diagrams $D_{T}, D_{P}$, which are identical outside $B$. In other words, $D_{P}$ is obtained by an $F_{o}$ move from the diagram $D_{T}$, where $D_{T}$ is a virtual link diagram by joining the end points of $T$. We will compute $Q_{D_{T}}(t)-Q_{D_{P}}(t)$. Let $I_{t}$ be the ideal of $\mathbb{Z}\left[t^{ \pm 1}\right]$ generated by these polynomials.

Outside $B$, there are 6 ways of connecting the end points of $T$ or $P$. Then, it is easy to check that we have the following table by computing $Q_{D_{T}}(t)-$ $Q_{D_{P}}(t)$. For instance, in the case of outside connection [16][25][34], the virtual intersection indices of real crossings $a, b, a^{\prime}, b^{\prime}$ in Figure 8 are $i(a)=\alpha+1, i(b)=$ $\alpha+\beta-1, i\left(a^{\prime}\right)=\alpha, i\left(b^{\prime}\right)=\alpha+\beta$ for some $\alpha, \beta \in \mathbb{Z}$. Then we have

$$
\begin{aligned}
Q_{D_{T}}(t)-Q_{D_{P}}(t) & =\left(t^{\alpha+1}-1\right)+\left(t^{\alpha+\beta-1}-1\right)-\left(t^{\alpha}-1\right)-\left(t^{\alpha+\beta}-1\right) \\
& =(t-1)^{2} B(t)
\end{aligned}
$$

for some $B(t) \in \mathbb{Z}\left[t^{ \pm 1}\right]$. By the similar computation, in the case of outside connection [15][26][34], we have $Q_{D_{T}}(t)-Q_{D_{P}}(t)=(t-1)^{2} A(t)$ for some $A(t) \in \mathbb{Z}\left[t^{ \pm 1}\right]$.

Table 3

\begin{tabular}{|c|l|}
\hline outside connection & $Q_{D_{T}}(t)-Q_{D_{P}}(t)$ \\
\hline$[14][25][36]$ & 0 \\
\hline$[14][26][35]$ & 0 \\
\hline$[15][24][36]$ & 0 \\
\hline$[15][26][34]$ & $(t-1)^{2} A(t)$ \\
\hline$[16][24][35]$ & $(t-1)^{2} B(t)$ \\
\hline$[16][25][34]$ & 0 \\
\hline
\end{tabular}


Similarly, we obtain the same result for the other cases of $T$ and $P$ with different orientations.

Then $I_{t}$ is the principal ideal generated by $g(t)=(t-1)^{2}$ and the following result holds.

Theorem 3.5. If $D$ and $D^{\prime}$ are isotopic welded diagrams, then $Q_{D}(t) \equiv Q_{D^{\prime}}(t)$ $\bmod I_{t}$.

Proof. From the above calculations, $Q_{D}(t)-Q_{D^{\prime}}(t) \in I_{t}$.

Let $R$ denote the quotient $\operatorname{ring} \mathbb{Z}\left[t^{ \pm 1}\right] / I_{t}$.

Corollary 3.6. The index polynomial $Q_{D}(t)$ of an oriented welded link $D$ in $R$ is a welded isotopy invariant.

Now consider oriented tangles $T^{\prime}$ and $P^{\prime}$ obtained from $T$ and $P$ by changing all over crossings to under crossings. For the ideal $I_{t}^{\prime}$ generated by $Q_{D_{T^{\prime}}}(t)-$ $Q_{D_{P^{\prime}}}(t)^{\prime}$ 's for all possible $T^{\prime}$ and $P^{\prime}$, by a similar computation as before, $I_{t}=I_{t}^{\prime}$ holds. Therefore, Thus if we allow both $F_{o}$ moves and $F_{u}$ moves, we have the following.

Theorem 3.7. The index polynomial $Q_{D}(t)$ of fused links in $R$ is a fused isotopy invariant.

Proof. Since we have $I_{t}=I_{t}^{\prime}$, the result follows.

Since any virtual knot is fused isotopic to the unknot, we get the following result.

Theorem 3.8. The index polynomial $Q_{D}(t)$ of any virtual knot diagram $D$ in $R$ is 0 .

Proof. Since the index polynomial $Q_{D}(t)$ of the unknot is 0 , the result follows.

Corollary 3.9. If $D$ is virtual knot diagram, then $Q_{D}(t) \in \mathbb{Z}\left[t^{ \pm 1}\right]$ is divisible by $(t-1)^{2}$.

Finally, we give an example of non-trivial welded link.

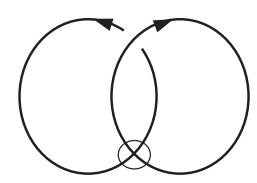

FiguRE 19. Virtual Hopf link

Example 3.10. Let $D$ be a welded link diagram as in Figure 19. Then it is immediate that $Q_{D}(t)=t^{-1}-1$. Thus, $Q_{D}(t)$ does not belong to the principal ideal $I_{t}$ and $D$ is a non-trivial welded link diagram. 


\section{References}

[1] A. Fish and E. Keyman, Jones polynomial invariants, J. Knot Theory Ramifications 15 (2006), no. 3, 339-350.

[2] R. Fenn, R. Rimányi, and C. Rourke, The braid-permutation group, Topology 36 (1997), no. 1, 123-135.

[3] M. Goussarov, M. Polyak, and O. Viro, Finite type invariants of classical and virtual knots, Topology 39 (2000), no. 5, 1045-1068.

[4] Y. H. Im, K. Lee, and S. Y. Lee, Index polynomial invariant of virtual links, J. Knot Theory Ramifications 19 (2010), no. 5, 709-725.

[5] Y. H. Im and K. I. Park, A parity and a multi-variable polynomial invariant for virtual links, J. Knot Theory Ramifications 22 (2013), no. 13, 1350073, 18 pp.

[6] Y. H. Im, K. I. Park, and M. H. Shin, Parities and polynomial invariats for virtual links, preprint.

[7] S. Kamada, Braid presentation of virtual knots and welded knots, Osaka J. Math. 44 (2007), no. 2, 441-458.

[8] N. Kamada and S. Kamada, Abstract link diagrams and virtual knots, J. Knot Theory Ramifications 9 (2000), no. 1, 93-106.

[9] N. Kamada and Y. Miyazawa, A 2-variable polynomial invariant for a virtual link derived from magnetic graphs, Hiroshima Math. J. 35 (2005), no. 2, 309-326.

[10] T. Kanenobu, Forbidden moves unknot a virtual knot, J. Knot Theory Ramifications 10 (2001), no. 1, 89-96.

[11] L. Kauffman, Virtual knot theory, European J. Combin. 20 (1999), no. 7, 663-690.

[12] Y. Miyazawa, Magnetic graphs and an invariant for virtual links, J. Knot Theory Ramifications 15 (2006), no. 10, 1319-1334.

[13] S. Nelson, Unknotting virtual knots with Guass diagram forbidden moves, J. Knot Theory Ramifications 10 (2001), no. 6, 931-935.

[14] S. Satoh, Virtual knot presentation of ribbon torus-knots, J. Knot Theory Ramifications 9 (2000), no. 4, 531-542.

[15] B. Winter, The classification of spun torus knots, J. Knot Theory Ramifications 9 (2009), no. 9, 1287-1298.

YOUNG Ho IM

Department of Mathematics

Pusan National University

PUSAN 609-735, KoreA

E-mail address: yhim@pusan.ac.kr

Kyeonghui LeE

Department of Mathematics

Pusan National University

Pusan 609-735, Korea

E-mail address: 1kh97@pusan.ac.kr

Mi HWA SHIN

Department of Mathematics

Graduate School of Natural Sciences

Pusan National University

PUSAN 609-735, KoreA

E-mail address: mhshin@pusan.ac.kr 